\title{
Effect of a radiation field on electrons bound on liquid helium
}

\author{
L. C. M. Miranda and R. M. O. Galvão \\ Centro Técnico Aeroespacial, Instituto de Estudos Avançados, 12200 São José dos Campos, \\ São Paulo, Brazil \\ C. A. S. Lima \\ Instituto de Física, Universidade Estadual de Campinas, 13100 Campinas, São Paulo, Brazil
}

(Received 20 June 1983)

\begin{abstract}
Using the image-potential model, we discuss the influence of a radiation field on the distortion of the bound states of an electron trapped on a liquid-helium surface. It is shown that, for moderate field strengths, the ionization energy decreases with increasing field strength.
\end{abstract}

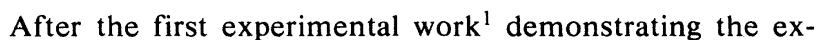
istence of a potential barrier to extract electrons from liquid helium, the interaction between electrons and a liquidhelium surface has been the subject of intense investigation in the last decade. ${ }^{1-10}$ The following description of the electron trapping is now generally accepted; an electron outside the liquid is attracted to it by the image force arising from the polarization of the surface, and is repelled by surface barrier as a result of the exclusion principle. The electron motion perpendicular to the surface of the liquid is thus quantized whereas along the liquid surface the transport is free.

The original works ${ }^{2-4}$ on the bound states of an electron trapped according to the above picture used a onedimensional hydrogenlike model for the binding potential. Even though the agreement between this model and the spectroscopic data on the transition energies between the ground state and the first two excited states was reasonably good, subsequent improvements of the energy separation calculations had taken into account the repulsive barrier of the surface. More recently, Hipolito, Felicio, and Farias, ${ }^{5}$ based upon the results on the image potential of a metalvacuum interface, ${ }^{4,6,7}$ have produced excellent agreement between theory and experiment with use of a modified image potential model. ${ }^{5}$ According to Refs. 4 and 5, and assuming that the liquid helium occupies the space $z<0$, the potential energy of the electron is given by

$$
V(z)=\left\{\begin{array}{l}
V_{0}, \quad z<0, \\
-\frac{Z e^{2}}{(z+\beta)}, \quad z>0,
\end{array}\right.
$$

where $V_{0}$ is the repulsive barrier potential, $Z$ is the strength of the image potential, namely, $Z=(\epsilon-1) / 4(\epsilon+1)$, where $\epsilon$ is the dielectric constant, and $\beta$ is the position of the center of mass of the induced charge. For liquid helium, $\epsilon=1.05723, V_{0}=1 \mathrm{eV}$, and the value of $\beta$ found in Ref. 5 was $1.01 \AA$. This value of $\beta$ is in very good agreement with the estimates of Grimes, Brown, Burns, and $\mathrm{Ziepfel}^{4}$ (i.e., $\beta=1.04 \AA$ ) using their experimental data.

In this paper, we extend the works of Refs. 4 and 5 by reporting on the effects of a radiation field on the bound states of electrons bound to the surface of liquid helium. The same problem has recently been studied by Jensen ${ }^{10}$ who show that this one-dimensional system is probably one of the best systems for the investigation of quantum stochasticity. The radiation beam is described by a classical plane electromagnetic wave of frequency $\omega$, linearly polarized along the normal to the liquid-helium surface (i.e., along the $z$ direction). The radiation is also assumed to be nonresonant with the zero-field system; i.e., we assume that the radiation frequency is smaller than the field-free binding energy. As the energies of our problem are of the order of $Z^{2} e^{2} / 2 a_{0}$ (or $6.6 \times 10^{-4} \mathrm{eV}$ ), we shall then assume a long wavelength radiation beam such as to a $10-$ to $50-\mathrm{GHz} \mathrm{mi}$ crowave. In the absence of the microwave field the electron trapping is assumed to be described by the image potential model of Eq. (1). Furthermore, we assume that the microwave field acts only on the electrons, having therefore no effect on the liquid-helium properties; i.e., we assume that both $\epsilon$ and $V_{0}$ are not changed by the presence of the microwave. Accordingly, in the presence of the radiation, the electron motion is described by the solution to the onedimensional Schrödinger equation $(Z<0)$

$$
i \hbar \dot{\psi}=\left[\frac{1}{2 m}\left(\hat{p}+\frac{e}{c} A(t)\right)^{2}+V(z)\right] \psi,
$$

where $\hat{p}=(\hbar / i) \partial / \partial z, A(t)=A \sin \omega t$ is the vector potential for the microwave beam in the dipole approximation, and $V(z)$ is given by Eq. (1).

To solve Eq. (2), we perform a unitary transformation, ${ }^{11-15}$ namely,

$$
\psi=e^{i \delta(t) \hat{p} / \hbar} e^{i \eta(t) / \hbar} \phi,
$$

where

$$
\delta(t)=-\frac{e}{m c} \int^{t} d t^{\prime} A\left(t^{\prime}\right)=\frac{e A}{m c \omega} \cos (\omega t)
$$

and

$$
\eta(t)=-\frac{e^{2}}{2 m c^{2}} \int^{t} d t^{\prime} A^{2}\left(t^{\prime}\right)
$$

We find

$$
i \hbar \dot{\phi}=\left(\frac{\hat{p}^{2}}{2 m}+V(z-\delta(t))\right) \phi .
$$

Equation (5) shows that in the presence of a laser field, the electron motion may be alternatively described by the Schrödinger equation for an electron moving in a potential whose center of force appears to be oscillating with the frequency $\omega$ and amplitude $e A / m c \omega$, namely, the dressed potential $V(z-\delta(t))$. Since we are assuming that the radia- 
tion field acts only upon the electron outside the surface, the explicit expression for the dressed potential is then

$$
V(z-\delta(t))=\left\{\begin{array}{l}
V_{0}, \quad z<0, \\
\frac{Z e^{2}}{\left[(z+\beta)^{2}+a^{2}-2 \sqrt{2}(z+\beta) a \cos (\omega t)+a^{2} \cos 2(\omega t)\right]^{1 / 2}}, \quad z>0,
\end{array}\right.
$$

where $a=e A / \sqrt{2} m c \omega$.

Equation (5) together with Eq. (6) has no exact analytical solution. Nevertheless, there is a wide range of radiation intensities and frequency for which some suitable approximate solution can be found. To see this let us rewrite Eq. (6b) as

$$
V(z-\delta)=-\frac{Z e^{2}}{\left[(z+\beta)^{2}+a^{2}\right]^{1 / 2}}(1-R)^{-1 / 2},
$$

where

$$
R=\frac{2 \sqrt{2}(z+\beta) a \cos (\omega t)-a^{2} \cos (2 \omega t)}{(z+\beta)^{2}+a^{2}} .
$$

As $R$ is smaller than one, we may expand Eq. (7) in a power series of $R$. The even terms in $R$ of this series give us time-independent (dc) terms, whereas the odd terms in $R$ leads to time-dependent (ac) terms. Collecting these two type of contributions, Eq. (7) can then be written as

$$
V=V_{\mathrm{dc}}+V_{\mathrm{ac}} \text {, }
$$

where

$$
\begin{aligned}
& V_{\mathrm{dc}}=-\frac{Z e^{2}}{\left[(z+\beta)^{2}+a^{2}\right]^{1 / 2}}\left\{1+\frac{3}{16} \frac{a^{4}+8 a^{2}(z+\beta)^{2}}{\left[(z+\beta)^{2}+a^{2}\right]^{2}}+\frac{105}{1024} \frac{a^{8}+32 a^{6}(z+\beta)^{2}+64 a^{4}(z+\beta)^{4}}{\left[(z+\beta)^{2}+a^{2}\right]^{4}}+\cdots\right\}, \\
& V_{\mathrm{ac}}=-\frac{Z e^{2}}{\left[(z+\beta)^{2}+a^{2}\right]^{1 / 2}}\left\{\frac{\sqrt{2} a(z+\beta) \cos (\omega t)}{\left[(z+\beta)^{2}+a^{2}\right]}-\frac{a^{2} \cos (2 \omega t)}{2\left[(z+\beta)^{2}+a^{2}\right]}+\cdots\right\} .
\end{aligned}
$$

We note that $V_{\text {dc }}$ is essentially the time average of the dressed potential. It is responsible for the appearance of the radiation-distorted bound states, whereas the ac terms cause the transitions between these states to take place, thereby limiting the range of radiation fields in which one can identify the dressed states. This is quite similar to the case of atoms in a laser field as discussed in Refs. 11-13.

To find the radiation-dressed bound states we have solved numerically the Schrödinger equation for the electron moving in the potential $V$ given by

$$
V= \begin{cases}V_{0}, & z<0, \\ V_{\mathrm{dc}}, & z>0 .\end{cases}
$$

In doing so, we have approximated $V_{\mathrm{dc}}$ by the successive terms of Eq. (8a). The results from our numerical calculations show that one can safely retain only the first two terms of Eq. (8a) to describe the main effects of the radiation field on the energy spectrum of the electron. For instance, the contribution of the third term of Eq. (8a) amounts to roughly $10 \%$ that of the first two terms. This means that the higher-order terms of $V_{\mathrm{dc}}$ are indeed small compared with the first two terms and can be treated as a perturbation to the first two terms. In Figs. 1 and 2 we plot, respectively, the dependence of the energy and of the transition frequencies of the three lowest states as a function of the radiation field strength for the case where $V_{\mathrm{dc}}$ is approximated by the first two terms of Eq. (8a). In our calculations the energies are measured in units of $Z^{2} \mathrm{Ry}$, and the lengths in units of the effective Bohr radius $\bar{a}_{0}=a_{0} / Z$. We have also introduced the dimensionless parameter $\lambda=a \sqrt{a_{0}}$ as a measure of the amplitude of the electron oscillation in units of $\bar{a}_{0}$. The parameter $\lambda$ is related to the radiation's electric field strength $E$ (in $V / \mathrm{cm}$ ) and frequency $f($ in $\mathrm{Hz})$ by

$$
E=1.68 \times 10^{-22} \frac{\lambda f^{2}}{Z}
$$

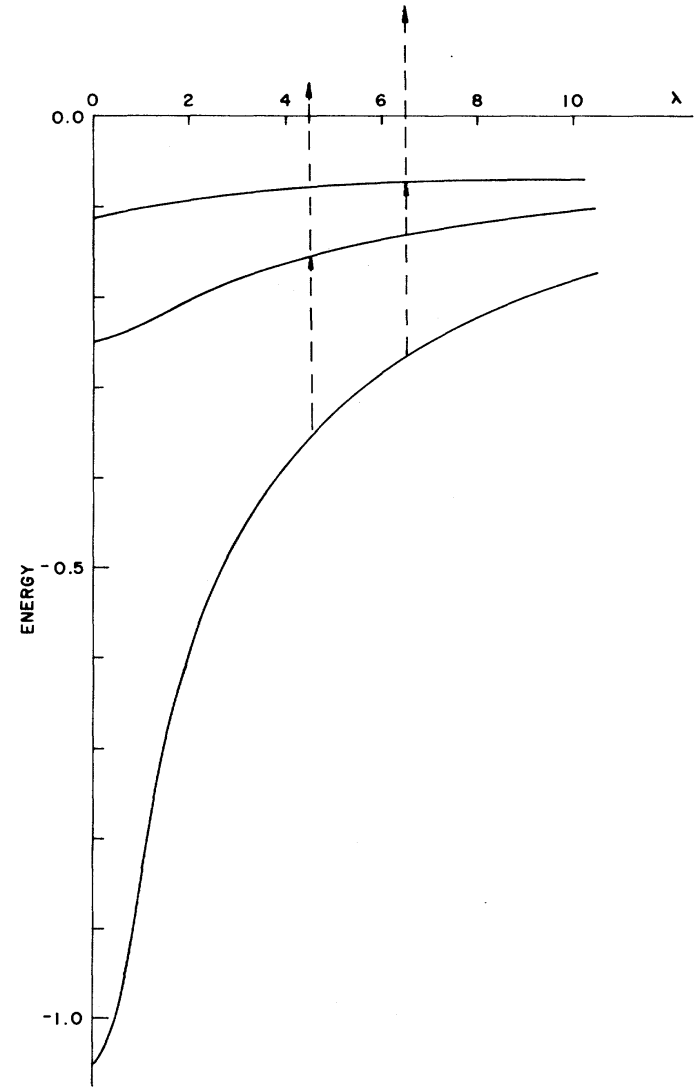

FIG. 1. Energy in units of $Z^{2} \mathrm{Ry}$, as a function of the electric field strength, for the three lowest states of the radiation-dressed image potential. $V_{\mathrm{dc}}$ is approximated by the first two terms of Eq. (8a) of the text. The dashed lines indicate the points at which the $1 \rightarrow 2 \rightarrow$ continuum and $1 \rightarrow 3 \rightarrow$ continuum transitions occur when using a $30-\mathrm{GHz}$ microwave as the distorting field. 


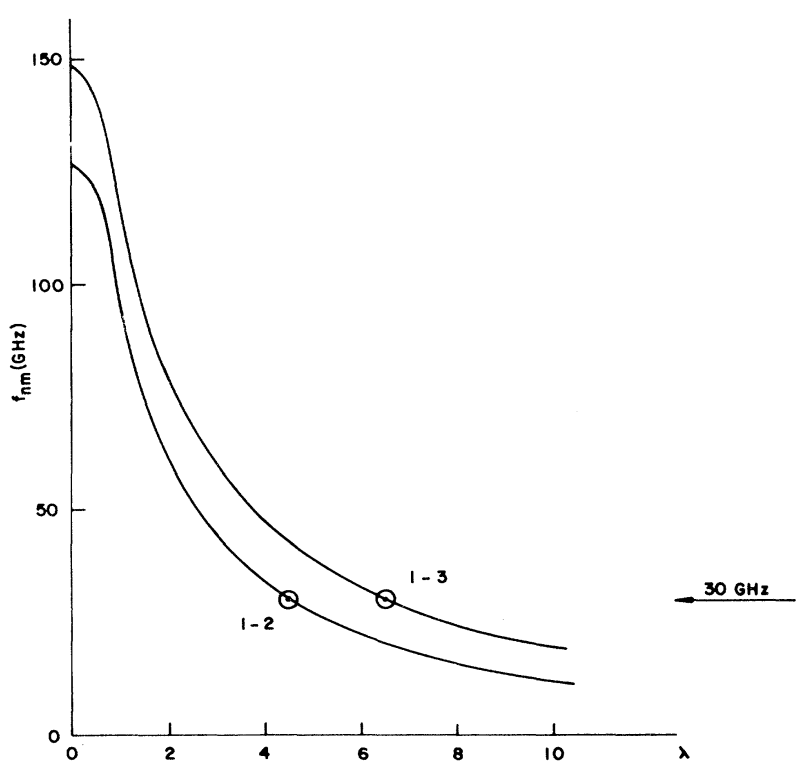

FIG. 2. Frequencies for the $1 \rightarrow 2$ and $1 \rightarrow 3$ transitions as a function of the radiation electric field strength. For $\lambda=0$ our results reproduce those of Ref. 5 .

Thus, for $Z=0.00695$ and $f=30 \mathrm{GHz}, \lambda=1$ corresponds to an electric field strength of roughly $22 \mathrm{~V} / \mathrm{cm}$.

It follows from Figs. 1 and 2 that, on increasing the radiation field strength $\lambda$, the bound-state energies decrease at the same time that the energy separation between successive states also decreases. This means that the effect of the radi- ation field points in the direction of a weakening of the electron binding such that, for very large values of $\lambda$, there is a tendency for the energy spectrum to become essentially continuous. Here, however, one should exercise some caution since a meaningful identification of these radiation-dressed states is possible only when multiphoton or single-photon transitions, due to the ac potential, are not taking place. In other words, long before one can reach large values of $\lambda$, the electron may be ionized by the multiphoton processes of Eq. (8b). To see this, let us consider the case where the radiation field is that of a $30-\mathrm{GHz}$ microwave. Referring to Fig. 1 we see that, on increasing the radiation field strength, one may eventually reach a point where the ground-state energy equals the radiation photon energy. For the case of a $30-\mathrm{GHz}$ microwave this happens (cf., Fig. 2) at $\lambda=4.5$, or for an electric field strength of $98 \mathrm{~V} / \mathrm{cm}$. At this point, on further absorbing another photon, the electron gets ionized. If, however, not all the electrons in the ground state get ionized by this cascade process at $\lambda=4.5$, on further increasing the electric field strength up to $141.6 \mathrm{~V} / \mathrm{cm}$ (or $\lambda=6.5$ ) the remaining electrons may be ionized by the two-photon sequence $1 \rightarrow 3 \rightarrow$ continuum, as indicated in Fig. 1.

To sum up, one may say that, in the presence of a radiation field, the electrons trapped by a liquid-helium surface acquire new radiation-dressed states such that the ionization energy decreases with increasing field strength. Furthermore, the identification of an electron in these dressed states is only possible in a range of field strengths such that there is no resonance between the field frequency and the dressed states. This range of field strengths is ultimately dictated by the driving frequency; for the case where a 30 $\mathrm{GHz}$ microwave is the driving field, the upper field strength is of the order of $98 \mathrm{~V} / \mathrm{cm}$.
${ }^{1}$ W. T. Sommer, Phys. Rev. Lett. 12, 271 (1964); M. A. Woolf and G. W. Rayfield, ibid. 15, 235 (1965); L. Bruschi, B. Maraviglia, and F. E. Moss, ibid. $1 \overline{7}, 682$ (1966).

${ }^{2}$ M. W. Cole and M. H. Cohen, Phys. Rev. Lett. 23, 1238 (1969).

${ }^{3}$ V. B. Shikin, Zh. Eksp. Teor. Fiz. 묘, 1748 (1970) [Sov. Phys. JETP 31, 946 (1970)].

${ }^{4}$ C. C. Grimes, T. R. Brown, M. L. Burns, and C. G. Ziepfel, Phys. Rev. B 13, 140 (1976); C. C. Grimes and T. R. Brown, Phys. Rev. Lett. 32, 280 (1974).

${ }^{5}$ O. Hipolito, J. R. D. Felício, and G. A. Farias, Solid State Commun. 28, 365 (1978).

${ }^{6}$ N. D. Lang and W. Kohn, Phys. Rev. B 7, 3541 (1973).

${ }^{7}$ J. Heinrichs, Phys. Rev. B $\underline{8}, 1346$ (1973).

${ }^{8}$ A. M. Gabovich, L. G. Ilchenko, and E. A. Pashitskii, Zh. Eksp.
Teor. Fiz. 79, 665 (1980) [Sov. Phys. JETP 52, 336 (1980)]; $\underline{81}$, 2063 (1981) [54, 1089 (1981)].

${ }^{9}$ Yu. P. Monarkha and V. B. Shikin, Fiz. Nizk. Temp. $\underline{8}, 563$ (1982) [Sov. J. Low Temp. Phys. $\underline{8}, 279$ (1982)].

${ }^{10}$ R. V. Jensen, Phys. Rev. Lett. 49, 1365 (1982).

${ }^{11}$ C. A. S. Lima and L. C. M. Miranda, Phys. Rev. A 23, 3335 (1981).

${ }^{12}$ T. C. Landgraf, J. R. Leite, N. S. Almeida, C. A. S. Lima, and L. C. M. Miranda, Phys. Lett. 92A, 131 (1982).

${ }^{13}$ N. S. Almeida and L. C. M. Miranda, Opt. Commun. $\underline{42}, 241$ (1982).

${ }^{14}$ R. M. O. Galvão and L. C. M. Miranda, Am. J. Phys. $\underline{51}, 729$ (1983).

${ }^{15}$ L. C. M. Miranda, Solid State Commun. 45, 783 (1982). 